\title{
Blazars at Low Radio Frequencies
}

\section{Jonas Trüstedt ${ }^{\star a b}$, Matthias Kadler ${ }^{a}$, Marcus Brüggen ${ }^{c}$, Heino Falcke ${ }^{e d}$, George Heald $^{d}$, John McKean ${ }^{d}$, Cornelia Müller $^{a b}$, Eduardo Ros ${ }^{f g h}$, Robert Schulz ${ }^{a b}$, Jörn Wilms $^{b}$}

${ }^{a}$ Lehrstuhl für Astronomie, Universität Würzburg, Campus Hubland Nord, Emil-Fischer-Str. 31, D-97074 Würzburg, Germany

${ }^{b}$ Dr, Remeis Sternwarte \& ECAP, Universität Erlangen-Nürnberg, Sternwartstr. 7, D-96049, Bamberg, Germany

${ }^{c}$ Universität Hamburg, Hamburger Sternwarte, Gojenbergsweg 112, D-21029, Hamburg, Germany

${ }^{d}$ ASTRON, The Netherlands Institute for Radio Astronomy, Postbus 2, 7990 AA, Dwingeloo, The Netherlands

${ }^{e}$ IMAPP/Department of Astrophysics, Radboud University, PO Box 9010, 6500 GL, Nijmegen, The Netherlands

${ }^{f}$ Max-Planck-Institut für Radioastronomie, Auf dem Hügel 69, D-53121 Bonn, Germany

${ }^{g}$ Observatori Astronòmic, Universitat de València, C. Catedrático José Beltrán 2, E-46980 Paterna, València, Spain

${ }^{h}$ Departament d'Astronomia i Astrofísica, Universitat de València, C. Dr. Moliner 50, E-46100 Burjassot, València, Spain

E-mail: jonas.truestedteastro.uni-wuerzburg.de

\begin{abstract}
We explore the low radio-frequency properties of the MOJAVE 1 blazar sample using the LOFAR Multi-Frequency Snapshot Sky Survey (MSSS). We find the characteristically flat blazar spectrum to extend down to the LOFAR bands, demonstrating that the emission at these low radio frequencies is still dominated by relativistically beamed emission. As most sources remain unresolved at the MSSS angular resolution, we are reimaging these data using LOFAR baselines beyond the standard MSSS uv-range resulting in an angular resolution of $\sim 24 \mathrm{arcsec}$. We present first LOFAR images of MOJAVE sources from this project.
\end{abstract}

12th European VLBI Network Symposium and Users Meeting

7-10 October 2014

Cagliari, Italy

\footnotetext{
* Speaker.
} 


\section{Introduction}

Active galactic nuclei (AGN) form jets due to accretion onto a supermassive black hole and eject them perpendicularly to their accretion disk. AGN jets have highly relativistic speeds, which is observed as apparent superluminal motion [1] if oriented at small inclination angles to the line of sight (these AGN are referred to as blazars). They extend out to kpc-scale lobes interacting with the intergalatic medium and regulate the evolution of their host galaxies and clusters [2]. Blazars can be subdivided into flat-spectrum radio quasars and BL Lac objects. The unification model [4] generally assumes, that these two subclasses are the relativistically beamed counterparts of FRI and FRII radio galaxies [3]. At low radio frequencies, their emission is expected to be dominated by unbeamed steep-spectrum synchrotron emission from the optically thin parts of their extended jets and lobes while at high radio frequencies, the beamed flat-spectrum core emission takes over. VLBI monitoring at GHz frequencies is used to observe the structural variability and evolution of the beamed inner jet region of AGN on parsec scales. MOJAVE is the largest long-term program monitoring extragalactic jets in the Northern Hemisphere, which contains a statistical complete sample of 135 radio-selected AGN (MOJAVE1) and was extended to about 250 AGN adding lowluminosity AGN, high synchrotron-peaked blazars, and other AGN types of special importance $[5,6]$. The MOJAVE project offers a large database and full kinematic information at parsec scales at $15 \mathrm{GHz}$ since 1994 (including Lorentz factors and jet-inclination angles).

At sufficiently low radio frequencies, any steep-spectrum lobe emission will eventually dominate over the flat-spectrum component. This prediction is challenged by Massaro et al. (2013) [7]. Their study on a sample of 697 blazars using the VLSS (74 MHz) [8] and NVSS (1.4 GHz) [9] catalogs demonstrates, that about $60 \%$ of blazars show flat spectra ${ }^{1}(|\alpha|<0.5)$, with $99 \%$ of the sources with spectral index $|\alpha|<0.9$. This result strongly suggests that the blazar spectra are still dominated by the beamed core emission. However, these data are affected by variability and limited angular resolution (see Table 1), which make it difficult to separate the core and lobe emission.

Previous studies $[10,11]$ have surveyed the MOJAVE1 sample with the VLA at $1.4 \mathrm{GHz}$ to study the kpc-scale emission of blazars in the $\mathrm{cm}$ band. In about $93 \%$ of all sources significant extended emission could be detected. More than $25 \%$ of the quasars have powers intermediate between FRI and FRII galaxies which confirms the studies of smaller samples $[12,13]$ and further challenges the unification model. Moreover, many BL Lac objects show morphologies typical for more powerful FRII jets.

A strong correlation was found between the apparent parsec-scale jet speeds and the beamed radiocore luminosity [1]. Its analytic power, however, is limited by the "inversion problem": both the beamed core luminosity and the apparent VLBI jet speed are affected by Doppler boosting and it is difficult to derive the source-intrinsic jet speeds and luminosities from it. By applying probabilistic arguments, however, it is possible to derive unique information about the distribution of Lorentz factors in a sample of AGN. Not only the beamed core luminosity from the inner jet but also the extended lobe emission is correlated with the apparent jet speeds on parsec scales [11]. The extended emission is therefore considered to be a good proxy for the jet kinetic power.

Arshakian et al. (2010) [14] used archival data to interpolate radio flux densities to $151 \mathrm{MHz}$. Assuming that the optically thin lobe emission is dominating over the core emission and the inner jet

\footnotetext{
${ }^{1}$ adopting the convention $S_{v} \propto v^{\alpha}$
} 


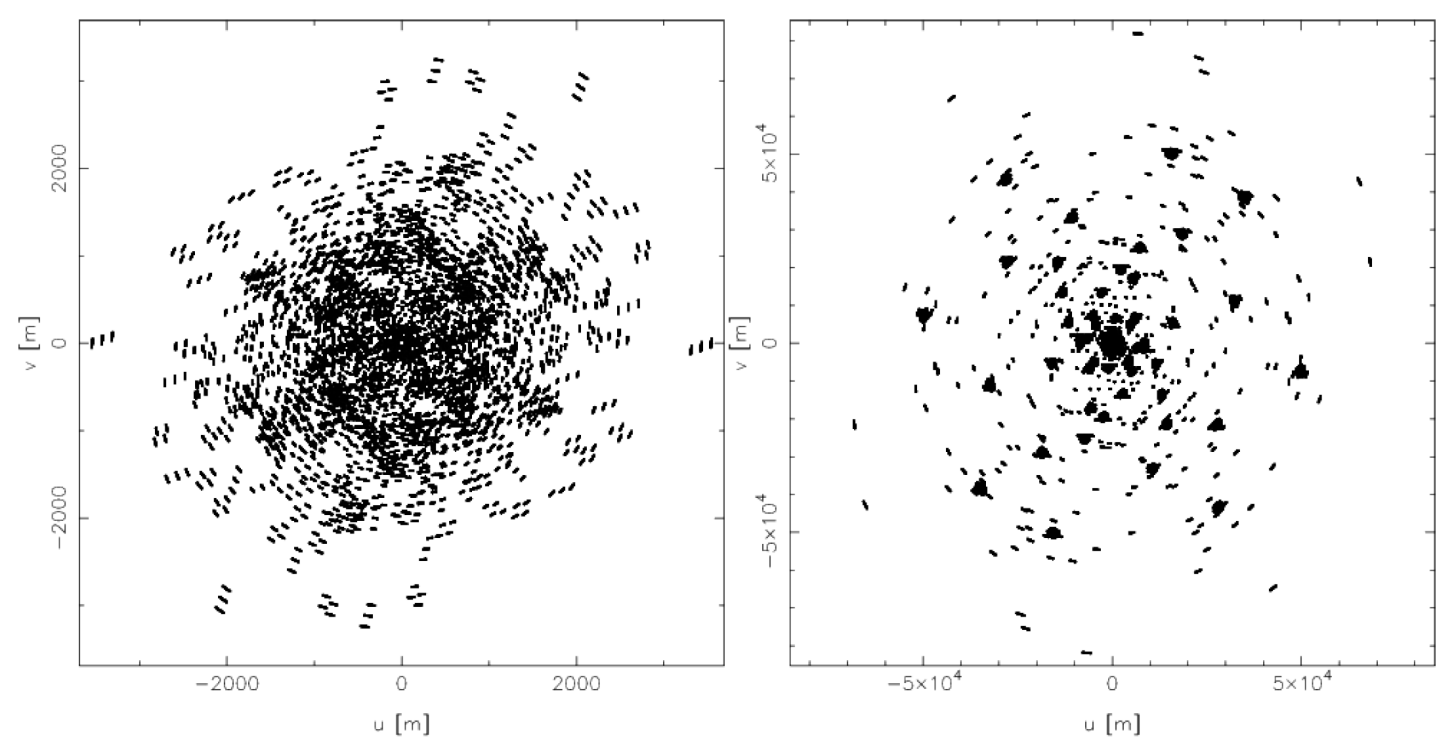

Figure 1: Example uv-coverage of one HBA field observation. On the left image only the data points of the core stations are plotted, while the right image includes all Dutch stations. Taken from [16]

region, they derive the intrinsic kinetic jet power. However, results by other studies [7] with VLSS data at $74 \mathrm{MHz}$ indicate that the emission is still beamed, but the resolution limits the possibility to draw conclusions on the extended emission. Being able to separate the beamed core emission from the extended oldest emission regions will offer the possibility to derive constraints on the Lorentz-factors. To do this, the flux-limited MOJAVE1 sample is a good candidate providing a large database on spectral properties and the apparent speeds of these sources.

\section{LOFAR and MSSS}

The Low Frequency Array (LOFAR) [15] offers the possibility to study blazars with high resolution at frequencies between $10-240 \mathrm{MHz}$. The array is centered in the Netherlands with outlying international stations in Germany, France, Sweden, and the United Kingdom. Every station consists of multiple dipoles. There are two different dipole types, the low-band antennas (LBA) covering the frequency range from $10 \mathrm{MHz}$ to $90 \mathrm{MHz}$ and the high-band antennas (HBA) covering the frequency range from $110 \mathrm{MHz}$ to $240 \mathrm{MHz}$. LOFAR is a key SKA pathfinder telescope [15] which will cover an even larger frequency bandwidth. One ongoing project of LOFAR is the multifrequency snapshot sky survey (MSSS) [16], which is designed to image the Northern Hemisphere and generate a catalog of all radio sources. This will be one of the first wide-area radio surveys with a large fractional bandwidth, which provides the opportunity to get spectral information on the studies by only using the survey data.

The MSSS observations are performed at 8 frequency bands around $50 \mathrm{MHz}$ with the low band antennas (LBA) and 8 bands around $135 \mathrm{MHz}$ with the high band antennas (HBA). Each frequency band has a bandwidth of $2 \mathrm{MHz}$. The observations consist of 9 snapshots of $11 \mathrm{~min}$ for the LBA frequency bands and 2 snapshots of $7 \mathrm{~min}$ for the HBA frequencies. All measurements also include polarization information. To image the complete northern sky the area is split into fields. This leads 


\begin{tabular}{cccc}
\multicolumn{4}{c}{ Table 1: Imaging capabilities } \\
Survey & Frequency (MHz) & Resolution $(\operatorname{arcsec})$ & Sensitivity $(\mathrm{mJy} / \mathrm{beam})$ \\
\hline Cooper/Kharb (VLA) [10, 11] & 1400 & 1.5 & 0.05 \\
NVSS (VLA) [9] & 1400 & 45 & 0.45 \\
VLSS (VLA) [8] & 74 & 80 & 100 \\
MSSS (standard) [16] & $120-160$ & $\sim 110$ & 5 \\
MSSS (reimaged) & $120-160$ & $\sim 24$ & $\sim 5$ \\
LOFAR (international) & $120-160$ & $<1$ & $<0.1$
\end{tabular}

to 660 fields for the LBA and 3616 fields for the HBA observations. These fields are combined into mosaics for each frequency band. Additionally, the 8 bands of each antenna are averaged into two images with full bandwidth of $16 \mathrm{MHz}$, one for LBA and one for HBA observations. When all mosaics are complete, the catalog of all sources in this survey will be generated. For more information see the MSSS survey description [16].

For the frequencies around $135 \mathrm{MHz}(\mathrm{HBA})$ the observations are already done and preliminary data products are available inside the MSSS team. Ongoing studies include research on variable sources and transients, magnetism, the search for pulsars, studies on galaxy clusters and star-forming galaxies, AGN, supernovae, unknown diffuse emission and the search for new supernova remnants and pulsar wind nebulae.

The preliminary HBA images are limited to a $(u, v)$-range of $2 \mathrm{k} \lambda$. For an example $(u, v)$-coverage see Fig. 1. This results in a resolution of about $110 \mathrm{arcsec}$ for the fields. A reprocessing of all MSSS data taking into account the longer-baseline data from all Dutch LOFAR stations is currently being done. While a theoretical resolution of 5 arcsec is possible, the large gaps in the $(u, v)$-coverage require further testing of the most feasible resolution. First results of this reprocessing achieve an angular resolution of about 24 arcsec, see Tab. 1.

\section{Preliminary results}

Our first results on the preliminary MSSS data confirm the earlier results [7], suggesting that emission of blazars at $\sim 135 \mathrm{MHz}$ is still dominated by beamed emission. In order to estimate the jet kinetic power, it is thus important to separate the core from the extended lobe emission. We used the reprocessed MSSS data, improving the angular resolution from $\sim 110 \operatorname{arcsec}$ to $\sim 24 \operatorname{arcsec}$ (see Table 1). However, not all of the sources show extended emission on these scales consistent with the previous MOJAVE results $[10,11]$ at $1.4 \mathrm{GHz}$ from VLA observations.

Besides measuring the intrinsic jet power, the observations can help to extend the studies of the MOJAVE team $[10,11]$ on the question of the large-scale structure of blazars and the BL Lac/Quasar vs FR I/II classification schemes in the AGN unification model. Figure 2 shows two examples for the reimaged MSSS data using the full $(u, v)$-coverage available. We find substantial extended lobe emission in the BL Lac object 3C 371 which is reminiscent of an FR I morphology seen face on. The quasar $4 \mathrm{C}+31.63$ shows extended emission suggesting a double sided jet. This is in agreement with the structure seen with the VLA at $1.4 \mathrm{GHz}$ [10], although the reprocessed MSSS data still do not reach the same angular resolution.

In order to compare the $135 \mathrm{MHz}$ structures of AGN with $1.4 \mathrm{GHz}$ VLA observations and search for the oldest extended emission, higher resolution and sensitivity are needed, which can be achieved 
by using international baselines in deeper LOFAR observations. Such observations have been started in 2014 and are planned to be continued in the upcoming LOFAR cycles.

\section{Acknowledgments}

J.T., C.M. and R.S. wish to thank RadioNet3 for support to attend this meeting.

\section{References}

[1] Cohen, M. H. et al., Relativistic Beaming and the Intrinsic Properties of Extragalactic Radio Jets, ApJ 658232 (2006)

[2] McNamara, B. R. et al., Heating Hot Atmospheres with Active Galactic Nuclei, ARA\&A 45117 (2007)

[3] Fanaroff, B. L. et al., The morphology of extragalactic radio sources of high and low luminosity, MNRAS 16731 (1974)

[4] Urry, C. M. et al., Unified Schemes for Radio-Loud Active Galactic Nuclei, PASP 107803 (1995)

[5] Lister, M. L. et al., $\gamma$-Ray and Parsec-scale Jet Properties of a Complete Sample of Blazars From the MOJAVE Program, ApJ 74227 (2011)

[6] Lister, M. L. et al., MOJAVE. X. Parsec-scale Jet Orientation Variations and Superluminal Motion in Active Galactic Nuclei, AJ 146120 (2011)

[7] Massaro, F. et al., Blazar Spectral Properties at 74 MHz, ApJS 20815 (2013)

[8] Cohen, A. S. et al., The VLA Low-Frequency Sky Survey, AJ 1341245 (2007)

[9] Condon, J. J. et al., The NRAO VLA Sky Survey, AJ 1151693 (1998)

[10] Cooper, N. J. et al., MOJAVE: Monitoring of Jets in Active Galactic Nuclei with VLBA Experiments. III. Deep VLA Images at 1.4 GHz, ApJS 171376 (2007)

[11] Kharb, P. et al., Extended Radio Emission in MOJAVE Blazars: Challenges to Unification, ApJ 710 764 (2010)

[12] Landt, H. et al., On the relationship between BL Lacertae objects and radio galaxies, MNRAS 391 967 (2008)

[13] Rector, T. A. et al., The Properties of the Radio-Selected 1 Jy Sample of BL Lacertae Objects, AJ 122 565 (2001)

[14] Arshakian, T. G. et al., Radio-optical scrutiny of compact AGN: correlations between properties of pc-scale jets and optical nuclear emission, A\&A 520 A62 (2010)

[15] van Haarlem, M. P. et al., LOFAR: The LOw-Frequency ARray, A\&A 556 A2 (2013)

[16] Heald, G. H. et al., The LOFAR Multifrequency Snapshot Sky Survey (MSSS), A\&A submitted 

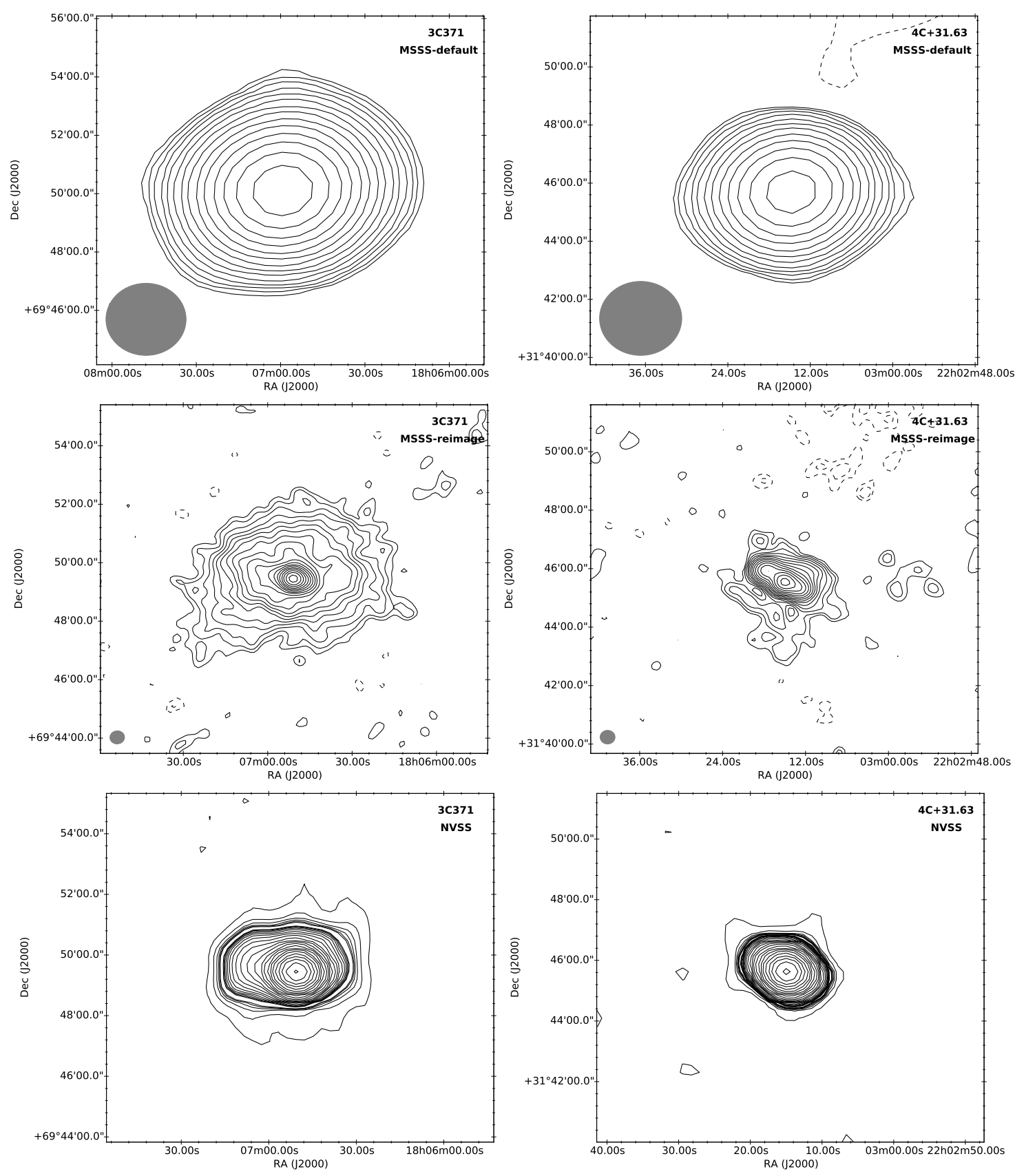

Figure 2: Example of preliminary MSSS default images at $\sim 135 \mathrm{MHz}$ (top), reimaged MSSS-data at $\sim 135$ $\mathrm{MHz}$ (middle) compared to NVSS images at $1.4 \mathrm{GHz}$ (bottom). MSSS images are the results of averaging over all 8 bands of the HBA data. 